\title{
Effect of FAC Chemotherapy on Plasma Insulin Concentration
}

\author{
Alkhansa Salih ${ }^{1}$, Khalid Hussein Bakheat ${ }^{2}$, Mohammed E A ${ }^{3,4}$ \\ ${ }^{1}$ Sudan Atomic Energy Commission- Khartoum- Sudan \\ ${ }^{2}$ Faculty of medicine- University of Khartoum- Khartoum-Sudan \\ ${ }^{3}$ Sudan Atomic Energy Commission- Khartoum- Sudan \\ ${ }^{4}$ Department of chemistry- Faculty of Science- King Khalid University-Saudi Arabia
}

\begin{abstract}
:
Introduction: 5-Fluorouacil, Adriamycin (doxorubicin) and Cyclophosphamide (FAC) is one of the major chemotherapy options for breast cancer.
\end{abstract}

Objective: the aim of this article is to investigate the effect of FAC chemotherapy on the blood insulin concentration.

Methods: 28 breast cancer patients and 28 normal subjects were involved in this study after informed consent. $5 \mathrm{mls}$ of intravenous blood samples were obtained from the control subjects and from the patients at diagnosis time (before starting chemotherapy) and after chemotherapy. The insulin concentration was measured using Immuno Radio Metric Assay (IRMA).

Results: all the study subjects were females and their age ranges were 26- 65. Half of study subjects received 3 cycles of FAC therapy and the rest received 6 cycles. The insulin concentration range, mean and standard deviation of the control subjects, patients before receiving the therapy and patients after receiving the therapy were (4- 16.8, 9.74 and 3.74), (4.1- 56.7, 17.53 and 11.04) and (5.6- 58, 22.94 and 11.31). However, 12 of the patients were with high insulin concentration (hyperinsulinemia) compared to the control subjects and the FAC therapy increased the number of patients with high insulin concentration to 21 . When the mean of insulin of the control subjects was compared to the means of insulin in the patients pre and post treatment, it was clear that breast cancer was associated with significant increase in insulin concentration since the p-value was 0.001 and the FAC therapy significantly increased the insulin concentration ( $p$-value $\leq 0.000)$. When the mean of insulin concentration in the patients before treatment was compared to its mean after FAC therapy, the p-value was equal to 0.057.

Conclusion:12 (42.9\%) of the newly diagnosed breast cancer patientswere associated with hyperinsulinemia and the FAC therapy increased the number of patients with hyperinsulinemia to 21 (75\%) i.e. the FAC therapy increased the insulin concentration.

Key words: FAC therapy, Insulin, IRMA, Sudanese, breast cancer patients.

\section{Introduction}

One of the major chemotherapy options for cancers is the FAC. FAC is a combination of three drugs; 5-Fluorouracil, Adriamycin (doxorubicin) and Cyclophosphamide.They are widely used as an antineoplastic agents which could be prescribed in combination or separately in most of malignancies including breast cancer [1]. The chemical structures of FAC components are shownbelow.

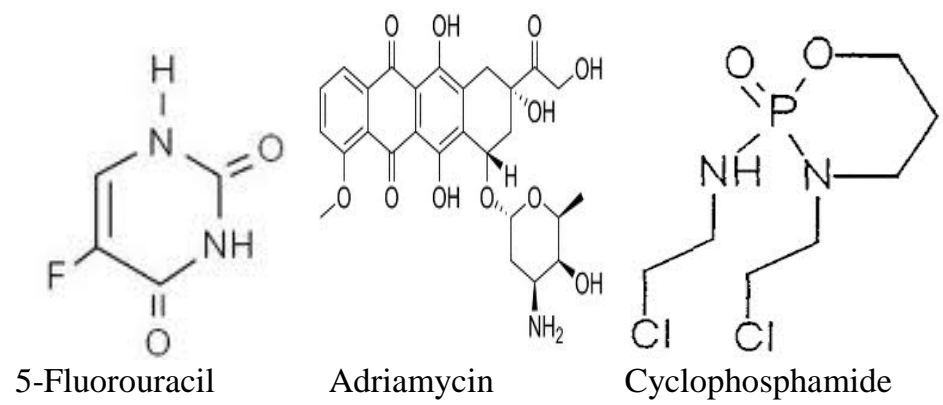

Regarding the mechanism of action of FAC constituents, 5 Fluorouracil is metabolized to Fluorodeoxyuridine mono phosphate which in turn binds covalently to thymidylatesynthetase and inhibits formation of deoxy thymidine tri phosphate. Inhibition of thymidine tri phosphate synthesis leads to inhibition of DNA synthesis and finally the arrest of cell division. Moderate inhibition of RNA synthesis is registered due to incorporation of flurouracil in the structure of RNA[2,3]. Adriamycin arrests cell division through inhibiting 
DNA replication. It stops DNA replication by inhibiting the topoisomerase II leading to abnormal removal of super twisting and sealing processes[4,5]. However, cyclophosphamide leads to cell deathby binding the DNA, interfering with mitosis and cell replication and inhibition protein synthesis[6,7].

The severity of 5-Fluorouracil, Adriamycin andCyclophosphamide side effects varies from person to person. Some of the common side effects include, Nausea, vomiting[8], loss of appetite, loss of hair, mouth sores, diarrhea, skin darkening[9].

Hyperinsulinemiais seen in people with early stageof diabetes milletus type 2, congenital hyperinsulinism and drug induced hyperinsulinemia and there is strong associaion between hypertension, dyslipidemia, obesity and glucose intolerance [10]. Most of the hyperinsulinemia symptoms are due to hypoglycemia which include temporary muscle weakness, brain fog, fatique, temporary inability to concentrate, visual problems such as blurred or double vision, headache, shaking and thirst [11].

\section{Objective}

The objective of this article is to investigate the effect of 5-Fluorouracil, Adriamycin and Cyclophosphamide chemotherapy (FAC) on serum insulin level in the blood of Sudanese breast cancerpatients.

\section{Study population}

\section{Material And Methods}

28newly diagnosed breast cancer patients were involved in this study after informed consent and they were followed after they received FAC therapy. The patients were compared to 28age and sex matched healthy subjects as controls. The patients were divided to two groups, 14 patients received three cycles of FAC and 14 patients received six cycles of FAC chemotherapy.

\section{Sampling}

$5 \mathrm{ml}$ venous blood sample was collected from each subjects in plain vacationer tube and left for serum separation. The serum was decanted and stored at $-20^{\circ} \mathrm{C}$.

\section{Methods}

Radioimmunoassay (RIA) technique was used to measure serum insulin level. RIA kits were supplied by the Department of Isotopes, China Institute of Atomic Energy and the assay was done according to the manufacturer instructions.

\section{Results}

All the study subjects (56) were females; 28 of them were healthy (control) and 28 were breast cancer patients and their age range was 26- 65. The patients were divided to two groups; group one received 3 cycles of FAC and group two received 6 cycles of FAC. However, the two groups contain 14 patients each.

The results of the insulin concentration in the blood of the control subjects and the patientspre and post chemotherapy are presented in table.1. However, when the mean of insulin concentration of the control subjects (9.67) was compared to its mean in the patients (17.46) before chemotherapy, the p- value was 0.001 i.e. breast cancer significantly increased the concentration of insulin. The patients who were with high insulin concentration compared to the control subjects were $12(42.9 \%)$ and the rest of the patients were with normal insulin concentration.

When the mean of the insulin concentration in the patients after chemotherapy (22.77) was compared to the mean of insulin in the control subjects $(9.67)$, the $p$ - value was $\leq 0.000$ i.e. the chemotherapy significantly increased the insulin concentration. The patients with high insulin concentration increased from 12 (before receiving FAC therapy) to 21 due to FAC therapy.

By comparing the mean of insulin concentration in the patients before FAC therapy (17.46) to the insulin mean concentration in the patients after FAC therapy (22.77), the p- value was 0.057 (Table.1). Twenty patients registered increased insulin concentration after FAC therapy and the FAC therapy caused decrease in insulin concentration in 8 patients.

As mentioned before, this study divided the patients into two groups; group one received 3 cycles of FAC (14) and group two received 6 cycles of FAC (14). By comparing the means of insulin level in the two groups (17.07 and 17.98 respectively) the p-value was 0.3 which means that 6 cycles of FAC insignificantly increased the insulin concentration (Table.2). 
Table.1: Ranges and means values of insulin in the patients pre and post treatmentcompared to the control subjects

\begin{tabular}{|l|l|l|l|l|l|}
\hline \multicolumn{2}{|c|}{} & Number & Insulin range & Insulin mean & $\mathrm{p}$ - value \\
\hline Control subjects & 28 & $4.0-16.8$ & 9.67 & \\
\cline { 1 - 5 } Patients & Pre & 28 & $4.1-56.7$ & 17.46 & \multirow{2}{*}{0.057} \\
\cline { 2 - 5 } & Post & 28 & $5.6-58$ & 22.77 & \\
\hline
\end{tabular}

Table.2: Comparison of the ranges and mean values of blood insulin
breast cancer patients who received 3 and 6 cycles of FAC chemothentration in the
\begin{tabular}{|l|l|l|l|l|}
\hline & Number & Insulin range & Insulin mean & p-value \\
\hline & 14 & $7-58$ & 17.07 & \\
\hline Patients who received 3 cycles of FAC & 14 & $5.6-34.5$ & 17.98 & 0.30 \\
\hline Patients who received 6 cycles of FAC &
\end{tabular}

\section{Discussion}

Concerning the newly diagnosed breast cancer patients 12 were found with high insulin concentration while 16 were with normal insulin concentration compared to the control subjects. However, when the means of insulin concentration of the control subjects and of the newly diagnosed patients were compared, the insulin concentration was significantly increased in the blood of the patients $(\mathrm{p}=0.001)$ i.e. breast cancer is associated with increased insulin concentration. However, Ian F Godsland and his colleagues in 2010 [12] stated that hyperinsulinemia may induce pro carcinogenic changes.Qiu-Li Zhu and his research team in 2010 [13] concluded that hyperinsulinemia induces malignant cells growthand it is associated with poor prognosis of breast cancer.Unlike our finding Autier P, 2013 and his colleagues [14] found that there is no association between insulin concentration and the risk for breast cancer

The insulin level post treatment was significantly increased in the patients compared to the control subjects $(\mathrm{p}<0.000) .21$ patients were found with high insulin level compared to the controls compared to 12 in the pretreatment group. However, when the post treatment mean insulin level was compared to its mean before treatment, it was clear that the FAC therapy (slight significantly) increased the insulin concentration (p- value $=0.057)$.It is known that chemotherapy induces changes in tumor biomarkers including insulin [12]. A Gualberto and his colleagues in stated, in the British Journal of cancer 2011 [15], that high ratio of insulin to IGFBP-1(Insulin like Growth Factor Binding Protein- 1) was predictive of the clinical benefit from F (Figitumumab) therapy to Non-Small-Cell Lung Cancerpatients (NSCLC).

In this study we have registered that 6cycles FAC chemotherapy insignificantly increased the level of insulin compared to three cycles of FAC, the same finding is expressed by Ian F Godsland and his colleagues [12].

This study concluded that $42.9 \%$ of the newly diagnosed breast cancer patients were associated with elevated insulin concentration and the FAC chemotherapy significantly increased the insulin concentration.

\section{References}

[1]. Rossella P, Massimo B, Simona G, Christopher AJ. Hydrophilic interaction liquid chromatography-APCI-mass spectrometry determination of 5-fluorouracil in plasma and tissues. J. Pharm. Biomed. Anal. 2005, 38: 738-745.

[2]. Parnes, C. Cirrincione, J. Aisner, D.A. Berry, S.L. Allen, J. Abrams, E. Chuang, M.R. Cooper, M.C. Perry, D.B. Duggan,T.P. Szatrowski, I.C. Henderson, and L. Norton.Phase III Study of Cyclophosphamide, Doxorubicin, and Fluorouracil (CAF) Plus Leucovorin Versus CAF for Metastatic Breast Cancer: Cancer and Leukemia Group B 9140.J Clin Oncol.2003, 21:1819-1824.

[3]. Shah MY, Pan X, Fix LN, Farwell MA, Zhang B. 5-Fluorouracil drug alters the microRNA expression profiles in MCF-7 breast cancer cells. J Cell Physiol. 2011, Jul; 226(7):1868-78.

[4]. Minotti G, Menna P, Salvatorelli E, Cairo G, Gianni L. Anthracyclines: molecular advances and pharmacologic developments in antitumor activity and cardiotoxicity. Pharmacol Rev 2004; 56: 185-229.

[5]. Zhou QY, Chowbay B. Determination of doxorubicin and its metabolites in rat serum and bile by LC: application to preclinical pharmacokinetic studies. J. Pharm. Biomed. Anal. 2002, 30: 1063-1074.

[6]. AmerAwad and Olaf Stüve. Cyclophosphamide in Multiple Sclerosis: Scientific Rationale, History and Novel Treatment Paradigms. TherAdvNeurolDisord. $2009,2(6): 50-61$.

[7]. MillyE de J, Selma MD, Michel JX, Hilde R, Alwin DR, Sjoerd R, Jos HB. Simultaneous quantification of cyclophosphamide, 4-hydroxycyclophosphamide, $\quad \mathrm{N}, \mathrm{N}$,, ',- triethylenethiophosphamide (thiotepa) and N,N',N"triethylenephosphoramide (tepa) in human plasma by high-performance liquid chromatography coupled with electro spray ionization tandem mass spectrometry (LC-MS/MS). J. Mass Spectr.2004, 39: 262-271.

[8]. Jassem J, Pieńkowski T, Płuzańska A, Jelic S, Gorbunova V, Mrsic-Krmpotic Z, Berzins J, Nagykalnai T, Wigler N, Renard J, Munier S, Weil C; Central \& Eastern Europe and Israel Pacitaxel Breast Cancer Study Group. Doxorubicin and paclitaxel versus fluorouracil, doxorubicin, and cyclophosphamide as first-line therapy for women with metastatic breast cancer: final results of a randomized phase III multicenter trial. J ClinOncol. 2001, 15; 19(6):1707-15.

[9]. CHARLES L. S HAPIRO and A BRAM R, ECHT, Side effects of adjuvant treatment in breast cancer, N Engl J Med, 2001, Vol. 344, No. 26.

[10]. Modan, Michaela, Halkin H, Almog S, Lusky A, Eshkol A, Shefi M, Shitrit A, \& Fuchs Z. Hyperinsulinemia: A link between hypertension obesity and glucose intolerance. J. Clin. Invest. 1985; 75 (3): 809-817.

[11]. Diabetes.co.uk. Hyperinsulinemia. Accessed at http://www.diabetes.co.uk/hyperinsulinemia.html on 3/3/2014 at 9:32 PM. 
[12]. Ian F Godsland. Insulin resistance and hyperinsulinemia in the development and progression of cancer. Clinical Science 2010; 118: 315- 332.

[13]. Qiu-Li Zhu, Wang-Hong Xu, Meng-Hua Tao. Biomarkers of the Metabolic Syndrome and Breast Cancer Prognosis. Cancers 2010; 2: 721-739.

[14]. Autier P, Koechlin A, Boniol M, Mullie P, Bolli G, Rosenstock J, Boyle P, Serum insulin and C-peptide concentration and breast cancer: a meta-analysis. Cancer causes control 2013; 24(5):873-83.

[15]. A Gualberto, ML Hixon, DD Karp, D Li, S Green, M Dolled-Filhart, LG Paz-Ares, S Novello, J Blakely,CJ Langer and MN Pollak Pre-treatment levels of circulating free IGF-1 identify NSCLC patients who derive clinical benefit from figitumumab. British Journal of Cancer (2011) 104, $68-74$. 